\title{
Polarization of Photoelectrons Ejected by Unpolarized Light from Xenon Atoms
}

\author{
U. Heinzmann, G. Schönhense, and J. Kessler \\ Physikalisches Institut der Universität Münster, D-4400 Münster, Germany
}

(Received 19 March 1979)

\begin{abstract}
The theoretical prediction that polarized photoelectrons can be ejected from unpolarized atoms by unpolarized radiation has been experimentally verified for xenon atoms. The wavelength dependence of the polarization has been measured and compared with theoretical results at helium and neon resonance wavelengths between 74.4 and $46.1 \mathrm{~nm}$.
\end{abstract}

In the experiments in which the spin polarization of photoelectrons has been studied, either the target or the radiation used for photoionization was usually polarized ${ }^{1}$ Spin polarization of photoelectrons that are ejected by unpolarized light from unpolarized targets has first been found with lead atoms last year ${ }^{2}$ after such an effect had been predicted theoretically for certain types of atoms. ${ }^{3.4}$ It is the purpose of this Letter to report detailed experimental results for xenon atoms and to compare the measured wavelength dependence of the polarization with theoretical predictions.

In order to observe the polarization one must not, as one would in the case of photoionization by circularly polarized light, detect all the photoelectrons, independent of their angle of emission. Instead, one has to detect photoelectrons emitted into a well-defined direction $\theta$. The situation is similar to electron scattering, in which electrons scattered through some angle $\theta$ become polarized. In both cases, the spin-orbit interaction is responsible for the polarization effect and the direction of the polarization is, for reasons of mirror symmetry, perpendicular to the reaction plane. If we let $\hat{k}_{i}$ and $\hat{k}_{0}$ be unit vectors in the directions of the incoming and the outgoing beam forming an angle $\theta$, according to theory ${ }^{3,4}$ the polarization of the photoelectrons may be written as

$$
\overrightarrow{\mathbf{P}}(\theta)=\frac{2 \xi \cos \theta}{1-\frac{1}{2} \beta\left(\frac{3}{2} \cos ^{2} \theta-\frac{1}{2}\right)} \hat{k}_{i} \times \hat{k}_{0} .
$$

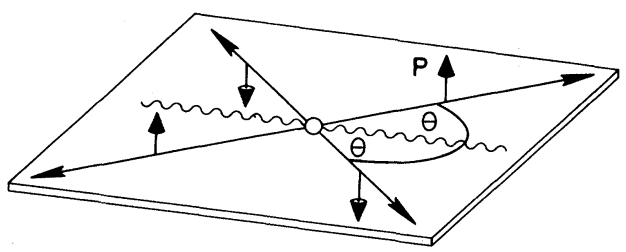

FIG. 1. $\pi$ periodicity of $P(\theta)$ when the result does not depend on whether the photon comes from the left or from the right. Any rotation by $\pi$ which transforms these two photon directions into one another must yield the same experimental result.
To get some insight into this equation we recall that the denominator in a polarization formula is proportional to the total number of electrons emerging into the direction of observation. ${ }^{1}$ The denominator in Eq. (1) is therefore proportional to the well-known expression for the angular distribution of the photoelectrons, $\beta$ being the asymmetry parameter.

Equation (1) holds in the (nonrelativistic) limit where the photon momentum is negligible compared with the photoelectron momentum. In this approximation the $\theta$ dependence of the polarization has period $\pi$ (see Fig。1) which is responsible for the term $\sin \theta \cos \theta$ in the denominator $[\mathrm{Eq}$. (1)]. The parameter ${ }^{5} \xi$ is determined by interference of the wave functions of those continuum states which are simultaneously reached by the photoionization process ( $s$ and $d$ states for photoionization of the $p$ shell). Like $\beta$, it depends on photon energy, photoelectron energy, and target atom. Its determination is a necessary step on the way to the complete photoionization experiment. ${ }^{6}$ The complication of such polarization measurements is that they combine the intensity problem of photoelectron spin analysis with the difficulties of photoelectron angular-distribution experiments.

A schematic diagram of the apparatus is shown in Fig. 2. As sources of vuv radiation we utilized capillary discharge tubes yielding intense helium and neon resonance lines (He I : $2 \times 10^{12}$ photons/s at the target). The angles between the radiation emitted by the two sources and the direction of

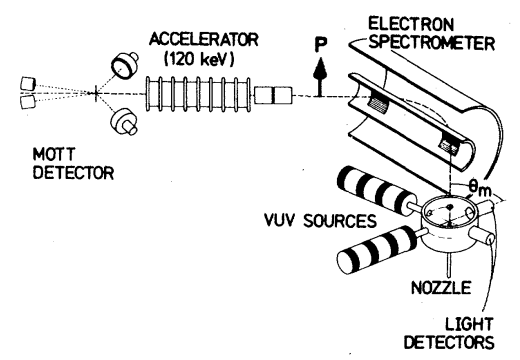

FIG. 2. Schematic diagram of the apparatus. 
the photoelectrons observed are $\theta_{m}-54^{\circ} 44^{\prime}$ (magic angle) and $180^{\circ}-\theta_{m}$. Since at these angles the denominator in $E q$ 。 (1) is 1, the polarization of the photoelectrons observed,

$$
P= \pm 2 \xi \sin 54^{\circ} 44^{\prime} \cos 54^{\circ} 44^{\prime},
$$

yields directly the parameter $\xi$ 。

The photoelectrons are produced in a xenon atomic beam and enter an electron spectrometer ${ }^{7}$ (cylindrical mirror analyzer) with an energy resolution of $0.7 \%$ (but not smaller than $35 \mathrm{meV}$ ) full width at half maximum. Thus we take account of the fact that at each wavelength photoelectrons of two distinct energies are produced; they correspond to the states ${ }^{2} P_{1 / 2}$ and ${ }^{2} P_{3 / 2}$ of the residual xenon ion, differing in energy by $1.3 \mathrm{eV}$. The photoelectrons with the energy selected are injected into an accelerator tube for $120 \mathrm{keV}$ and hit the gold foil of a Mott detector for polarization analysis. The polarization is determined from the left-right asymmetry of the intensity scattered into the two counters at $120^{\circ} ._{1}$ The ratio of true to background counts varied between 1000 and 1 because of large intensity differences of the various resonance lines and because of the cross-section differences. Typical background count rates were 10 counts/min. Instrumental asymmetries could easily be eliminated by taking advantage of the reversal of the polarization when switching from one light source to the other [see Eq. (1) or Eq. (2)]. The two counters at small angles allow additional corrections for instrumental asymmetries to be made. ${ }^{8}$

Figure 3 shows the polarizations obtained for various wavelengths or photoelectron energies and the values of $\xi$ resulting from these data. The polarizations of photoelectrons associated with the ${ }^{2} P_{1 / 2}$ and ${ }^{2} P_{3 / 2}$ states of the residual ion differ in sign (the situation shown in Fig. 1 corresponds to positive ${ }^{5} \xi$ ). This shows the necessity of resolving the fine structure in such a measurement. Otherwise the polarizations would almost cancel one another as predicted by Cherepkov $_{0}{ }^{4}$ The different sign of the polarization of the photoelectrons connected with the ${ }^{2} P_{1 / 2}$ and ${ }^{2} P_{3 / 2}$ ionic states can be qualitatively explained as follows: The $p_{1 / 2}$ electrons of the xenon atom reach the $d_{3 / 2}$ continuum leaving a ${ }^{2} P_{1 / 2}$ ion behind, whereas the electrons reaching the $d_{5 / 2}$ continuum leave behind a ${ }^{2} P_{3 / 2}$ ion. Since as a result of spinorbit interaction spin is not a good quantum number in the $d$ states, the $d$ wave functions are linear combinations of the two basic spin states. The linear combinations have different signs for
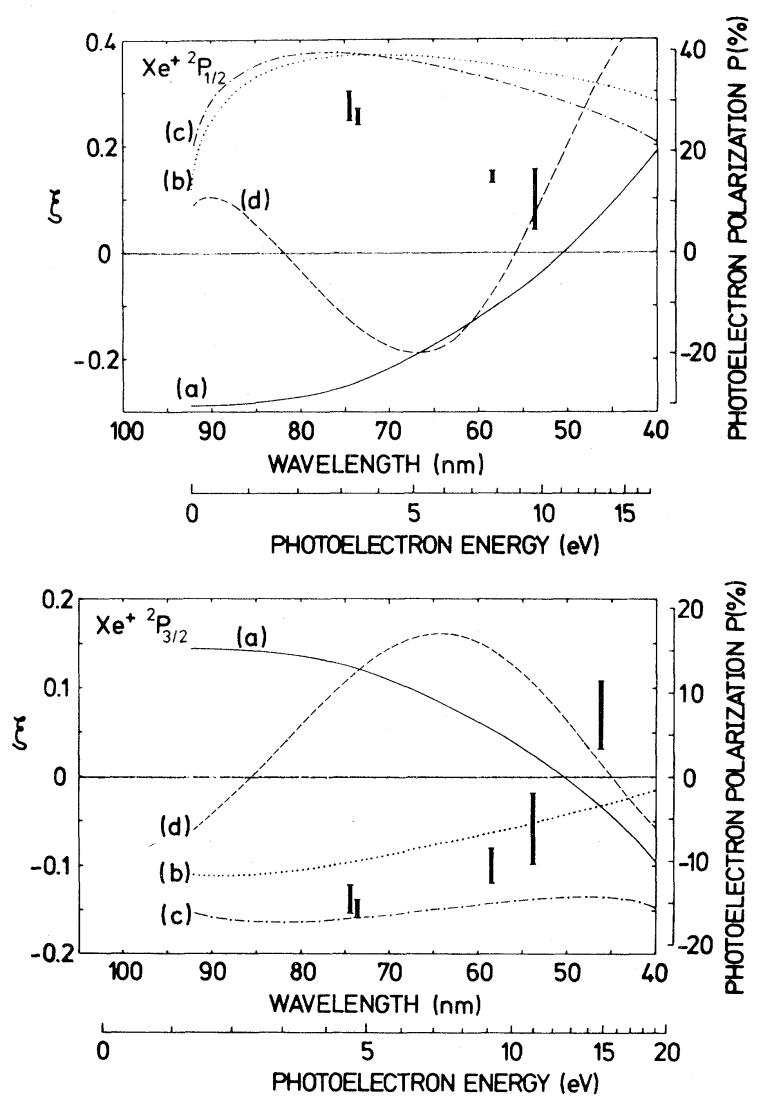

FIG. 3. Data points with error bars, experimental values ( 1 standard deviation) for photoelectron polarization (scale on the right) and for parameter $\xi$ (scale on the left). The curves follow from calculations using the random-phase approximation (curve $a$ ) or multichannel quantum-defect theory with different sets of parameters (curves $b-d$ ).

$d_{3 / 2}$ and $d_{5 / 2}$. This causes different signs of the $s-d_{3 / 2}$ and $s-d_{5 / 2}$ interference terms, which are the origin of the polarization, so that the polarizations connected with the ${ }^{2} P_{1 / 2}$ and ${ }^{2} P_{3 / 2}$ states of the residual ion differ in sign。

The error bars are mainly determined by the statistical error of the polarization measurement and include the uncertainty of the analyzing power (Sherman function)。 They differ from each other because of the large differences between the ratios of true to background counts. The correction for the angular divergence of $\pm 5.5^{\circ}$ of the photoelectrons accepted by the spectrometer turns out to be negligible. It is crucial for the reliability of the $\xi$ data given that there are no residual fields in the ionization chamber which may deflect the photoelectrons, thus leading to a spurious angle of emission. In order to check whether residual fields had been sufficiently suppressed 
we measured a great number of asymmetry parameters $\beta$ with our apparatus at the same wavelengths used for the polarization experiment. The agreement of our $\beta$ values for several noble gases and molecules with those found by other authors ${ }^{9}$ confirmed the reliability of the $\xi$ measurements.

Figure 3 shows also a few theoretical results. Curve $a$ calculated by Cherepkov using the random-phase approximation with exchange gives, for the case ${ }^{2} P_{3 / 2}$, the correct wavelength for the zero of $\xi$. The fact that this curve has the wrong sign although there is reasonable agreement with the absolute values of our measurements prompted us to review the correctness of the sign given by our polarization data. The ratio of the polarizations for the two states of the residual ion is expected ${ }^{4}$ to be -2 if the radial parts of the continuum wave functions for the $d_{3 / 2}$ and $d_{5 / 2}$ photoelectrons are the same. The experimental results indicate that the ratio differs from -2 , which shows that the influence of spin-orbit interaction on the continuum wave functions (the origin of the Fano effect ${ }^{10}$ ) cannot be neglected. The other curves of Fig. 3 have been calculated by means of "multichannel quantum-defect theory," with use of various sets of parameters that we found in the literature. In this theory, the wavelength dependence of $\xi$ is determined by that of the Coulomb phase shifts, the quantum defects, and the matrix elements for photoionization. For curves $b$ and $c$ we used the data of Lee and Dill ${ }^{11}$ and Geiger, ${ }^{12}$ respectively, with energy-independent quantum defects, whereas for curve $d$ we utilized the set of energy-dependent parameters of Geiger. ${ }^{13}$ In all three cases we have extrapolated the linear energy dependence of the data from the discrete to continuum energies.

Since the main purpose of this paper is to present the experimental results, details of the quantum-defect calculations, together with a set of quantum-defect parameters which give the best fit to the experimental data, will be given elsewhere.

We would like to thank Professor Dr. J. Geiger for several interesting discussions about the quantum-defect theory and acknowledge the assistance of A. Wolcke in setting up the computer system for evaluating the measured data. We also acknowledge support by the Deutsche Forschungsgemeinschaft.

${ }^{1} \mathrm{~J}$. Kessler, Polarized Electrons (Springer, Berlin, Heidelberg, New York, 1976).

${ }^{2}$ U. Heinzmann, G. Schönhense, and A. Wolcke, in Abstracts of Contributed Papers and Proceedings of the International Workshop on Coherence and Correlation in Atomic Collisions, University College, London, 1819, September 1978, edited by H. Kleinpoppen, and J. F. Williams (Plenum, New York, to be published). ${ }^{3}$ C. M. Lee, Phys. Rev. A 10, 1598 (1974).

${ }^{4}$ N. A. Cherepkov, Zh. Eksp. Teor. Fiz. 38, 933 (1974) [Sov. Phys. JETP 38, 463 (1974)], and J. Phys. B 11, L435 (1978).

${ }^{5}$ Unfortunately different authors do not use the same definition of $\xi$; our definition of $\xi$ is given by Eq. (1) which was given by Lee (Ref. 3).

${ }^{6}$ M. Ya. Amusia, Comments At. Mol. Phys. $\underline{8}, 61$ (1979).

${ }^{7}$ D. Brandt, Diplomarbeit, Universität Mümster, 1975 (unpublished).

${ }^{8}$ U. Heinzmann, J. Phys. B 11, 399 (1978).

${ }^{9}$ See, for example, J. L. Dehmer, W. A. Chupka, J. Berkowitz, and W. T. Jivery, Phys. Rev. A 12, 1966 (1975); D. L。 Miller, J。 D。 Dow, R. G. Houlgate, G. V. Marr, and J. B. West, J. Phys. B 10, 3205 (1977), and references therein.

${ }^{10}$ U. Fano, Phys。Rev。 178,131 (1969).

${ }^{11}$ D. Dill, Phys。Rev. A 7, 1976 (1973).

${ }^{12} \mathrm{~J}$. Geiger, Z. Phys. A 2 76, 219 (1976).

${ }^{13} \mathrm{~J}$. Geiger, Z. Phys. A $\underline{282}, 129$ (1977). 